\title{
Clinical outcome score predicts the need for neurodevelopmental intervention after infant heart surgery
}

\author{
Andrew S. Mackie, MD, SM, ${ }^{\mathrm{a}, \mathrm{b}, \mathrm{c}}$ Gwen Y. Alton, RN, MN, ${ }^{\mathrm{c}, \mathrm{d}}$ Irina A. Dinu, PhD, ${ }^{\mathrm{b}}$ Ari R. Joffe, MD, ${ }^{\mathrm{a}, \mathrm{c}}$ \\ Stephen J. Roth, MD, MPH, ${ }^{\mathrm{e}}$ Jane W. Newburger, MD, MPH, ${ }^{\mathrm{f}}$ and Charlene M. T. Robertson, MD ${ }^{\mathrm{a}, \mathrm{d}}$
}

\begin{abstract}
Objective: Our goal was to determine if a clinical outcome score derived from early postoperative events is associated with 18- to 24-month Psychomotor Developmental Index (PDI) score among infants undergoing cardiopulmonary bypass surgery.
\end{abstract}

\begin{abstract}
Methods: We included infants aged $\leq 6$ weeks who underwent surgery during 2002-2006, all of whom were referred for neurodevelopmental evaluation at age 18 to 24 months. We excluded children with chromosomal abnormalities, hearing loss, cerebral palsy, or a Bayley III assessment. The prespecified clinical outcome score had a range of 0 to 7 . Lower scores indicated a more rapid postoperative recovery. Patients requiring extracorporeal membrane oxygenation were assigned a score of 7 .
\end{abstract}

Results: Ninety-nine subjects were included. Surgical procedures were arterial switch $(\mathrm{n}=36)$, Norwood $(n=26)$, repair of total anomalous pulmonary venous connection $(n=16)$, and other $(n=21)$. Four subjects had postoperative extracorporeal membrane oxygenation. Clinical outcome scores were highest in the Norwood group (mean $4.1 \pm 1.4)$ compared with the arterial switch group $(1.9 \pm 1.6)(P<.001)$, total anomalous pulmonary venous connection group $(1.6 \pm 2.0)(P<.001)$, and other group $(3.3 \pm 1.6, P=$ not significant $)$. A mean decrease in PDI of 10.9 points ( $95 \%$ confidence interval, $4.9-16.9 ; P=.0005$ ) was observed among children who had a clinical outcome score $\geq 3$, compared with those with a clinical outcome score $<3$. Time until lactate $\leq 2.0 \mathrm{mmol} / \mathrm{L}$ increased with increasing clinical outcome score $(P=.0003)$, as did highest 24-hour inotrope score $(P<.0001)$.

Conclusions: Clinical outcome scores of $\geq 3$ were associated with a significantly lower PDI at age 18 to 24 months. This score may be valuable as an end point when evaluating novel potential therapies for this high-risk population. (J Thorac Cardiovasc Surg 2013;145:1248-54)

Earn CME credits at

http://cme.ctsnetjournals.org

Supplemental material is available online.

Mortality from congenital heart disease has decreased significantly during the past 3 decades. ${ }^{1,2}$ This is particularly true for infants undergoing complex cardiac surgical procedures. $^{2}$ As a consequence, neurodevelopmental

From the Department of Pediatrics, ${ }^{\text {a }}$ School of Public Health, ${ }^{\mathrm{b}}$ University of Alberta, Edmonton, Alberta, Canada; Stollery Children's Hospital, ${ }^{\mathrm{c}}$ Edmonton, Alberta, Canada; Glenrose Rehabilitation Hospital, ${ }^{\mathrm{d}}$ Edmonton, Alberta, Canada; Lucile Packard Children's Hospital and Stanford University, ${ }^{\mathrm{e}}$ Palo Alto, Calif; and Children's Hospital Boston and Harvard University, ${ }^{\mathrm{f}}$ Boston, Mass.

Disclosures: Authors have nothing to disclose with regard to commercial support.

Received for publication Jan 16, 2012; revisions received March 23, 2012; accepted for publication April 13, 2012; available ahead of print Sept 10, 2012.

Address for reprints: Andrew S. Mackie, MD, SM, Division of Cardiology, Stollery Children's Hospital, 4C2 Walter C. Mackenzie Centre, 8440-112th St, NW, Edmonton, Alberta, Canada T6G 2B7 (E-mail: andrew.mackie@ualberta.ca). $0022-5223 / \$ 36.00$

Copyright (c) 2013 by The American Association for Thoracic Surgery http://dx.doi.org/10.1016/j.jtcvs.2012.04.029 morbidity has become an increasingly relevant outcome. However, neurodevelopmental outcomes are difficult to predict in early childhood, and interventions in infancy that may affect neurodevelopment are challenging to evaluate given a time lag between intervention and neurodevelopmental assessment that may be 2 years or longer. Many innovative therapies for infants undergoing cardiopulmonary bypass (CPB) surgery have been evaluated in recent years and will continue to evolve, yet selection of primary end points for clinical trials remains challenging. These issues speak to the potential value of surrogate outcomes that reliably predict neurodevelopmental sequelae and can be measured early in the postoperative course.

In pediatric patients undergoing cardiac surgery, several scoring systems have been developed that predict mortality. ${ }^{3,4}$ However, correlation with neurodevelopmental outcomes has not been a focus of publications to date. Mackie and colleagues ${ }^{5}$ developed a composite clinical outcome score for neonates undergoing complex aortic arch reconstruction that discriminated patients receiving intravenous postoperative tri-iodothyronine from those receiving placebo. This score is objective and measurable in intensive care units (ICUs) at no cost. Possible scores range from 0 (reflecting least morbidity) to 7 (reflecting death or use of postoperative mechanical circulatory support). 


\section{Abbreviations and Acronyms \\ $\mathrm{CPB}=$ cardiopulmonary bypass \\ ICU = intensive care unit \\ PDI = Psychomotor Developmental Index \\ LOS = length of stay \\ BSID-II = Bayley Scales of Infant Development, 2nd edition \\ MDI $=$ Mental Developmental Index \\ TAPVC $=$ total anomalous pulmonary venous connection}

However, the full range of potential scores was not observed among patients in that initial validation study. ${ }^{5}$ Therefore, a modification of this score was developed. The objective of our study was to examine the performance of this modified score. We hypothesized that the modified clinical outcome score would correlate with Psychomotor Developmental Index (PDI) score at age 18 to 24 months. A secondary objective of this study was to determine the correlation between this modified clinical outcome score and other important perioperative events, namely time to normalization of postoperative lactate, postoperative inotrope score, postoperative ICU length of stay (LOS), and total hospital LOS.

\section{METHODS \\ Design}

This study is part of a larger multiprovince inception cohort that was established to evaluate neurodevelopmental outcomes of infants aged $\leq 6$ weeks who undergo cardiopulmonary bypass surgery in western Canada. ${ }^{6}$ This inception cohort prospectively collected preoperative, intraoperative, and postoperative variables, as previously described. ${ }^{7}$

\section{Subjects}

Inclusion criteria were infants $\leq 6$ weeks of age who underwent cardiopulmonary bypass surgery at the Stollery Children's Hospital in Edmonton, Alberta, Canada, between January 2002 and February 2006, all of whom received multidisciplinary neurodevelopmental assessments including the Bayley Scales of Infant Development (BSID-II) ${ }^{8}$ through a developmental clinic with the exception of 1 child who was lost to follow-up. Developmental clinics were located in Edmonton and Calgary, Alberta; Regina and Saskatoon, Saskatchewan; Vancouver, British Columbia; and Winnipeg, Manitoba, Canada. We excluded children with known chromosomal abnormalities (eg, Trisomy 21 or deletion 22q11.2), cerebral palsy, or moderate to severe hearing loss, and those who had a Bayley III assessment rather than a BSID-II, because the two are not equivalent. ${ }^{9}$ The inception cohort follow-up had ethics board approvals from each site. All parents/ guardians provided written informed consent. The Health Research Ethics Board at the University of Alberta approved the study.

\section{Early Childhood Assessments}

Children were evaluated at age 18 to 24 months. Certified pediatric psychologists and psychometrists administered the BSID-II, ${ }^{8}$ yielding separate Mental Developmental Index (MDI) and PDI standardized scores. The MDI assesses memory, problem solving, number concepts, vocalization, language, and social skills. The PDI assesses gross motor and fine motor skills. The mean score for both the MDI and PDI is $100 \pm 15$. Pediatricians experienced in neurodevelopmental assessment examined each child for evidence of cerebral palsy or visual impairment, defined as corrected visual acuity in the better eye of $<20 / 60$. Hearing was evaluated by experienced certified pediatric audiologists in soundproof environments as has been described. ${ }^{6}$ Hearing loss was defined as binaural sensorineural hearing loss of more than $40 \mathrm{~dB}$ at any frequency from 250 to $4000 \mathrm{~Hz}$. Maternal education was determined by years of schooling.

\section{Clinical Outcome Score}

The original clinical outcome score developed by Mackie and colleagues ${ }^{5}$ is shown in Table 1 . The components of the clinical outcomes score were derived with the intent of using variables that could be recorded objectively and early in the postoperative course, that are clinically meaningful (eg, time to first extubation) and that reflect hemodynamic status. No subjects in our study or the original publication had a score of 5 or 6 . To improve the distribution of subjects across the range of possible scores (0 to 7), a modified clinical outcome score was developed (Table 1) using the same variables as the previous score, but with a modified score weighting. The score was calculated by adding the subscore of each individual component (range, 0 to 2 each). Subjects requiring postoperative extracorporeal membrane oxygenation were assigned a score of 7 , the highest possible score, to reflect the most severe morbidity.

\section{Inotrope Score}

The postoperative inotrope score was calculated as per Wernovsky and colleagues. ${ }^{10}$ The highest 24-hour postoperative inotrope score for a given patient was recorded for the purpose of correlation with the clinical outcome score.

\section{Lactate}

Serum lactate was measured postoperatively in the ICU with a frequency that was determined by the responsible intensivist. All blood gases analyzed in the ICU have a point of care lactate result, and are usually done during the first postoperative day at least every 4 hours, as previously described. ${ }^{11}$ The time interval between weaning from cardiopulmonary bypass to first lactate $\leq 2.0 \mathrm{mmol} / \mathrm{L}$ was measured in hours.

\section{Statistical Analysis}

Categorical data are presented as proportions. Continuous data are expressed as mean \pm standard deviation or median with interquartile range. One-way analysis of variance with Tamhane's multiple comparisons and Fisher exact test were used to compare surgical groups; Bonferroni correction was applied. Descriptive variables for outcomes were analyzed with Student $t$ test, $\chi^{2}$ test, and Fisher exact test. Correlations between clinical outcome score and other outcome variables were examined using Spearman rank order correlation coefficient. Subjects with a clinical outcome score of 6 or 7 were grouped together due to small sample size in these groups. Exploratory data analyses supported the use of multiple indicators for the clinical outcome score, with the lowest score being the reference category. Multiple linear regression analysis included clinical outcome score and any other potential predictor variables having $P<.10$ on univariate linear regression analysis, and after checking for multicolinearity. Potential predictor variables considered were birth weight, gestational age at birth, age at surgery (in days), year of surgery, number of preoperative days of ventilation, duration of cardiopulmonary bypass, duration of deep hypothermic circulatory arrest, lowest flow rate on cardiopulmonary bypass, palliative procedure (eg, Norwood) versus complete biventricular repair, mother's years of schooling, and antenatal diagnosis (yes/no). All analyses were 2-sided. Statistical analyses were performed using SPSS version 17 (2008; IBM Co, Armonk, NY) and SAS version 9.1 (2007; SAS Institute, Cary, NC), and R version 2.30 (2009; Institute for Statistics and Mathematics, Vienna University, Vienna, Austria). 
TABLE 1. Clinical outcome score and modified clinical outcome score

\begin{tabular}{|c|c|c|c|c|c|c|}
\hline \multirow[b]{2}{*}{ Clinical variable } & \multicolumn{3}{|c|}{ Clinical outcome score } & \multicolumn{3}{|c|}{ Modified clinical outcome score } \\
\hline & $\mathbf{0}$ & 1 & 2 & $\mathbf{0}$ & 1 & 2 \\
\hline Time until first negative fluid balance (d) & $\leq 3$ & $4-5$ & $\geq 6$ & $\leq 1$ & 2 & $\geq 3$ \\
\hline Time until sternal closure (d) & $\leq 2$ & $3-4$ & $\geq 5$ & $\leq 1$ & $2-4$ & $\geq 5$ \\
\hline Time until first extubation (d) & $\leq 4$ & $5-8$ & $\geq 9$ & $\leq 4$ & $5-8$ & $\geq 9$ \\
\hline
\end{tabular}

ECMO, Extracorporeal membrane oxygenation.

\section{RESULTS}

One hundred seventy-one infants met inclusion criteria. Of these, 25 (14.6\%) died, 19 were excluded (12 for chromosomal abnormalities, 6 for sensorineural hearing loss, 1 for cerebral palsy), and 1 was lost to follow-up. Twentyseven had a Bayley III rather than BSID-II assessment. The remaining 99 subjects were included in this analysis. Surgical procedures performed were: arterial switch $(n=36)$, Norwood stage $1(n=26)$, repair of total anomalous pulmonary venous connection (TAPVC) $(\mathrm{n}=16)$, and other $(n=21)$. The other group consisted of complete repair of interrupted aortic arch $(\mathrm{n}=10)$, truncus arteriosus $(n=3)$, and tetralogy of Fallot $(n=2)$; Blalock-Taussig shunt for tetralogy of Fallot with atrioventricular septal defect $(n=1)$; Blalock-Taussig shunt for atrioventricular septal defect and pulmonary atresia $(\mathrm{n}=1)$; Yasui procedure for tunnel-like left ventricular outflow obstruction and ventricular septal defect $(n=1)$; pulmonary artery band and atrial septectomy for double outlet right ventricle $(\mathrm{n}=1)$; resection of a right ventricular mass $(\mathrm{n}=1)$; and tricuspid valve repair with transannular right ventricular outflow patch for Ebstein's anomaly with pulmonary atresia $(n=1)$.

Preoperative, intraoperative, and postoperative variables are provided in Table 2 . The distribution of modified clinical outcome scores among these 99 subjects is shown in Figure 1. Four subjects had postoperative extracorporeal membrane oxygenation and therefore were assigned a score of 7 . Clinical outcome scores were highest in the Norwood group with a mean of $4.1 \pm 1.4$ compared with the arterial switch group with a mean of $1.9 \pm 1.6(P<.001)$ and the TAPVC group with a mean of $1.6 \pm 2.0(P<.001)$. Scores in the other group were $3.3 \pm 1.6$, not statistically significantly different from the Norwood, arterial switch, or TAPVC groups.

All three components of the clinical outcome score (Table 1) correlated with PDI on univariate analysis (data not shown). On multiple regression analysis, PDI scores were lower amongst patients with a clinical outcome score $\geq 3$ (Table E1 and Figure 2). After adjusting for age at surgery, a decrease in PDI score of 10.9 (95\% confidence interval 4.9-16.9) $(P=.0005)$ was found among subjects with a clinical outcome score $\geq 3$ compared with those with a score $<3$. There was a marginally significant change of 12.5 in the percent of variation $\left(R^{2}\right)$ in PDI scores explained by the clinical outcome score when added to a model consisting of age at surgery $(P=.051)$. There was a trend toward a lower MDI score of 5.8 (95\% confidence interval, -0.5 to 12.1$)(P=.068)$ among subjects with a clinical outcome score $\geq 3$ compared with those with a score $<3$, after adjusting for age at surgery and years of mother's education (Table E2).

It was not possible to include ICU LOS, total hospital LOS, or duration of preoperative ventilation in the multiple regression analysis, because these variables were highly correlated with the clinical outcome score. On univariate analysis these variables all had a lesser effect on PDI as did the clinical outcome score, reflecting an advantage of the clinical outcome score over these variables. For example, the percentage of variance in PDI score explained by total hospital LOS was $21.3 \%$. However, each additional 30 days in hospital was associated with a mean decrease in PDI score of 6.6, compared with a mean decrease of 10.9 with clinical outcome scores of 3 or higher.

Longer time to first lactate $\leq 2.0 \mathrm{mmol} / \mathrm{L}$ and highest postoperative inotrope score both had a positive correlation with the clinical outcome score $(P=.0003$ and $P<.0001$, respectively) (Figures 3 and 4). Postoperative length of ICU stay also had a positive correlation with clinical outcome score $(P<.0001)$ (Figure E1), as did total hospital LOS $(P$ $<$.0001) (data not shown).

\section{DISCUSSION}

We found that a clinical outcome score derived from the timing of postoperative events among infants aged $\leq 6$ weeks undergoing cardiopulmonary bypass surgery was associated with PDI score at age, 18 to 24 months, time to normalization of serum lactate, highest postoperative inotrope score, and with ICU and total hospital LOS. Clinical outcome scores were higher among subjects undergoing Norwood stage 1 palliation compared with those undergoing the arterial switch procedure or repair of TAPVC. The full range of potential scores was observed among the study population. Subjects were equally divided among those having low scores ( 0 to 2 ), reflecting a more rapid postoperative recovery, and those having a high score (3 to 7), reflecting greater postoperative morbidity.

PDI scores were significantly lower among subjects having a clinical outcome score $\geq 3$, compared with those with a score $<3$. The relationship between clinical outcome score and PDI was not linear, suggesting that there is a threshold effect of perioperative hemodynamic status on 
TABLE 2. Preoperative, intraoperative, and postoperative variables by type of infant cardiac surgery

\begin{tabular}{|c|c|c|c|c|}
\hline Variable & Norwood $(n=26)$ & Arterial switch $(n=36)$ & TAPVC repair $(n=16)$ & Other $(n=21)$ \\
\hline \multicolumn{5}{|l|}{ Preoperative } \\
\hline Age at surgery (d) & $10.3 \pm 8.8$ & $12.3 \pm 8.2$ & $17 \pm 15$ & $18.4 \pm 15.2$ \\
\hline Gestational age (wk) & $39.6 \pm 1.2$ & $38.9 \pm 1.3$ & $39.3 \pm 1.6$ & $39.1 \pm 1.4$ \\
\hline Duration of preoperative ventilation (d) & $8.2 \pm 8.7$ & $5 \pm 4$ & $2.5 \pm 4.5$ & $5.1 \pm 7.9$ \\
\hline \multicolumn{5}{|l|}{ Intraoperative } \\
\hline Duration of cardiopulmonary bypass (min) & $130 \pm 52^{*}$ & $141 \pm 50^{*}$ & $61 \pm 28$ & $121 \pm 50^{*}$ \\
\hline Duration of aortic cross-clamping (min) & $40 \pm 13$ & $67 \pm 23^{*}, \dagger$ & $36 \pm 21$ & $56 \pm 36^{*}$ \\
\hline Duration of DHCA $(\min )(n=62)$ & $24.2 \pm 13.5$ & $7.4 \pm 4.5^{*}, \dagger$ & $27.1 \pm 6.9$ & $39.2 \pm 26.3$ \\
\hline Lowest flow $(\mathrm{mL} / \mathrm{kg} / \mathrm{min})($ for $>10 \mathrm{~min})$ & $80.7 \pm 43.5$ & $113.7 \pm 27.8$ & $145.3 \pm 10.1$ & $113 \pm 38.5$ \\
\hline \multicolumn{5}{|l|}{ Postoperative } \\
\hline Time until lactate $\leq 2.0 \mathrm{mmol} / \mathrm{L}(\mathrm{h})$ & $21.3 \pm 16.5$ & $14.2 \pm 17.8$ & $17.0 \pm 14.8$ & $17.4 \pm 12.9$ \\
\hline Highest 24-h inotrope score & $21.5 \pm 9.9$ & $14.1 \pm 8.5$ & $14.6 \pm 12.9$ & $17.5 \pm 14.9$ \\
\hline Time until first negative fluid balance (d) & $2.1 \pm 1.5$ & $1.6 \pm .6$ & $1.6 \pm .8$ & $2.1 \pm .5$ \\
\hline Delayed sternal closure $(\mathrm{n}=52)$ & 26 & 10 & 2 & 14 \\
\hline Time until sternal closure (d) & $5.4 \pm 4.4$ & $1.2 \pm 2.6 \dagger$ & $1.1 \pm 2.6 \dagger$ & $2.7 \pm 2.5$ \\
\hline Time until first extubation (d) & $11.7 \pm 5.6$ & $7.4 \pm 6.4$ & $5.8 \pm 4.0$ & $8.6 \pm 4.3$ \\
\hline Total duration of postoperative ventilation $\ddagger$ (d) & $15.6 \pm 15.8$ & $8.4 \pm 6.5$ & $7.9 \pm 6.2$ & $11.2 \pm 8.9$ \\
\hline Total hospital length of stay $\S(d)$ & $52.4 \pm 55.6$ & $23 \pm 13.6$ & $17.9 \pm 11.4$ & $27.7 \pm 16.7$ \\
\hline Postoperative length of stay, ICU and ward (d) & $44.3 \pm 54.7$ & $17.2 \pm 11.9$ & $15 \pm 9.7$ & $22.3 \pm 17.3$ \\
\hline Mother's years of schooling & $13.2 \pm 1.8$ & $12.1 \pm 3.3$ & $12.1 \pm 3.3$ & $14.4 \pm 1.9$ \\
\hline Age at neurodevelopmental testing (mo) & $21.1 \pm 3.5$ & $20.7 \pm 2.8$ & $21.3 \pm 3.8$ & $21.9 \pm 2.9$ \\
\hline Mental Developmental Index & $86.2 \pm 17.3$ & $88.2 \pm 16.9$ & $83.5 \pm 20.2$ & $82.8 \pm 16.3$ \\
\hline Psychomotor Developmental Index & $77.5 \pm 18.6$ & $90.6 \pm 11.3$ & $85.2 \pm 12.5$ & $82.5 \pm 17.1$ \\
\hline Clinical outcome score & $4.1 \pm 1.4^{*}$ & $1.9 \pm 1.6 \dagger$ & $1.6 \pm 2.0$ & $3.3 \pm 1.6$ \\
\hline
\end{tabular}

Results are presented as mean \pm standard deviation unless otherwise specified. DHCA, Deep hypothermic circulatory arrest; ICU, intensive care unit; TAPVC, total anomalous pulmonary venous connection. *Difference from TAPVC at $P<.001$. $\dagger$ Difference from Norwood at $P<.001$. †Fifteen patients required reintubation and therefore had a total duration of postoperative ventilation that exceeded time until first extubation. §Includes pre- and postoperative days.

cerebral injury. It is not possible to conclude to what degree congenital brain abnormalities or preoperative insults contributed to lower PDI scores, relative to a difficult postoperative clinical course reflected by a high clinical outcome score. Although the Bayley scales are not an intelligence test, low scores do identify patients at risk for poor neurodevelopmental outcomes. ${ }^{12-15}$ The relationship between clinical outcome score with MDI did not reach

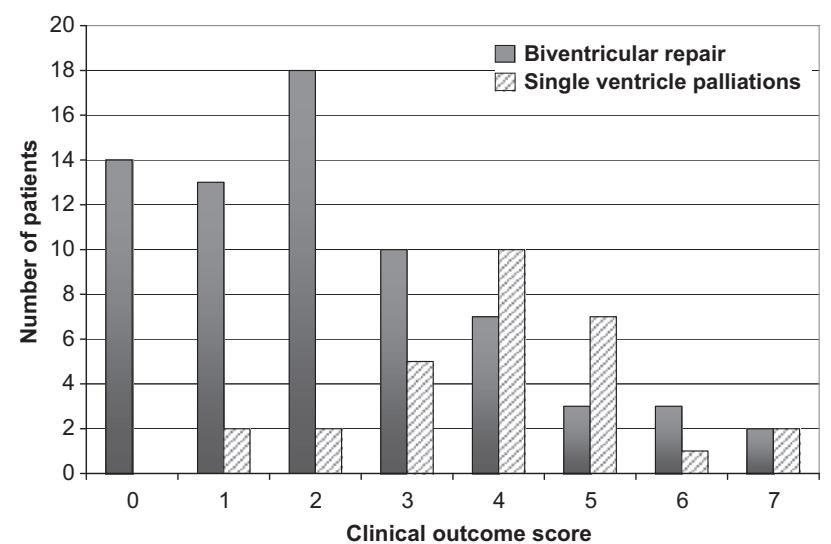

FIGURE 1. Distribution of modified clinical outcome scores among subjects undergoing a single ventricle palliative procedure $(n=29)$ (26 Norwood, 2 Blalock-Taussig shunt, and 1 pulmonary artery band with atrial septectomy) vs a biventricular repair $(\mathrm{n}=70)$. statistical significance. This may have occurred because MDI scores were less affected than PDI scores among the Norwood group, a finding that has been observed by others. $6,16,17$

Time until lactate $\leq 2.0 \mathrm{mmol} / \mathrm{L}$ and highest postoperative inotrope score reflect early postoperative hemodynamic status and correlated well with the clinical outcome score (Figures 3 and 4). Serum lactate has been used as a marker

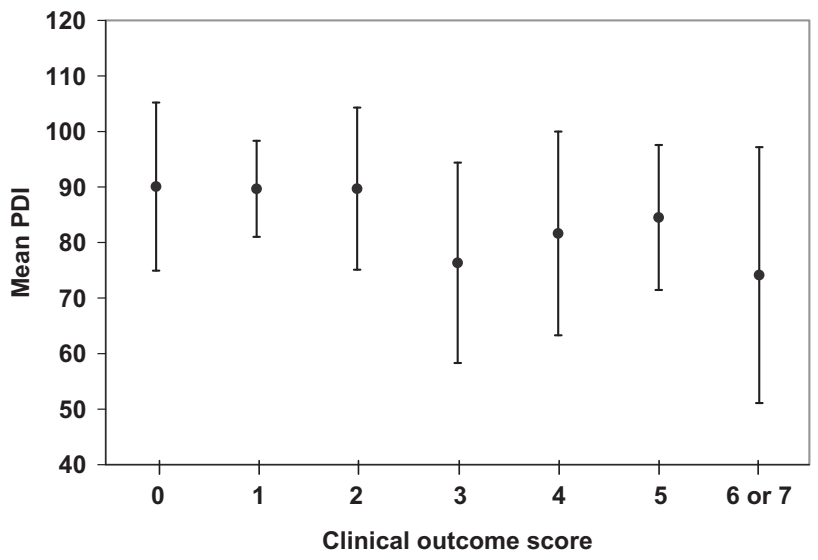

FIGURE 2. Relationship between mean Psychomotor Developmental Index $(P D I)$ score and clinical outcome score. Vertical bars represent 1 standard deviation. 


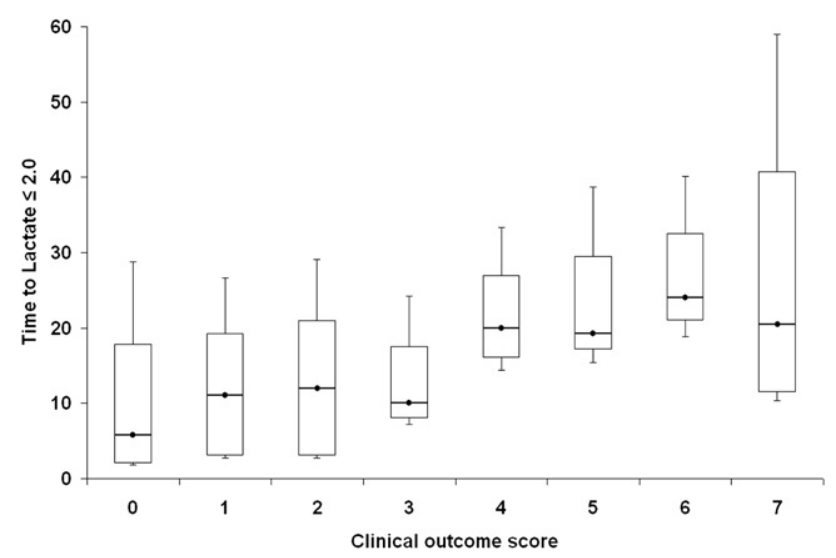

FIGURE 3. Relationship between postoperative time to lactate $\leq 2.0$ $\mathrm{mmol} / \mathrm{L}$ and clinical outcome score $($ rho $=0.36 ; P=.0003)$.

of adverse outcomes after pediatric cardiac surgery, including increased ICU LOS ${ }^{18}$ and mortality. ${ }^{11,18-20}$ Trittenwein and colleagues $^{21}$ demonstrated a relationship between higher lactate and cerebral damage after pediatric cardiac surgery, and our group demonstrated the value of serial postoperative lactate determination to help differentiate those with intact versus adverse neurodevelopmental outcome in a cross-sectional population after CPB surgery at age, $\leq 6$ weeks. ${ }^{11}$

Postoperative inotrope score has also been described as a surrogate for morbidity in children undergoing CPB surgery. Gaies and colleagues ${ }^{22}$ reported that a vasoactiveinotropic score modified slightly from the inotrope score originally described by Wernovsky and colleagues ${ }^{10}$ correlated strongly with time to first negative fluid balance, time to first extubation, and ICU LOS, and is consistent with our finding that the clinical outcome score correlated with highest 24-hour inotrope score. An association with neurodevelopmental outcome was not explored in the study by Gaies and colleagues ${ }^{22}$ and the predictive value of

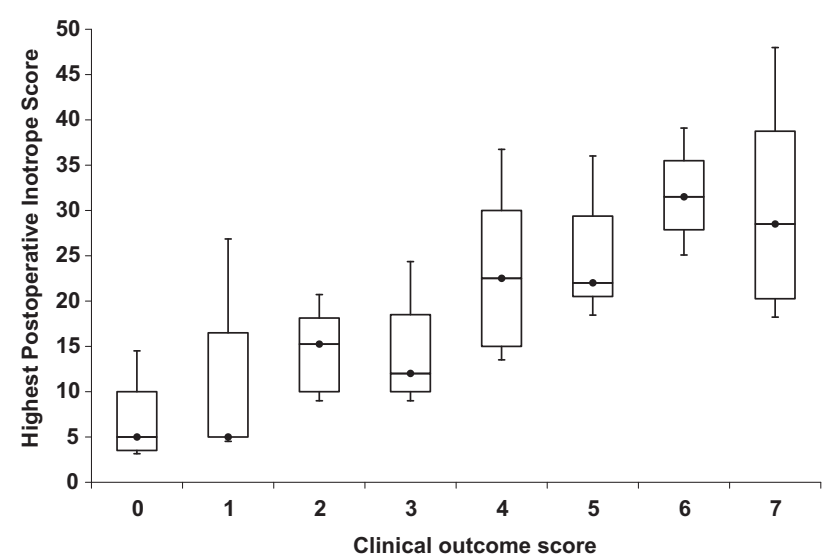

FIGURE 4. Relationship between highest postoperative inotrope score and clinical outcome score $($ rho $=0.61 ; P<.0001)$. inotrope scores relative to other postoperative parameters is unknown.

The correlation between clinical outcome score and ICU LOS (Figure E1) was anticipated given that the events determining the clinical outcome score (Table 1) must occur in the ICU rather than on the ward. However, the clinical outcome score also correlated well with total postoperative LOS (ICU and ward combined), which reflects the sum of both early and late postoperative morbidity. This is relevant as LOS correlates with neurodevelopmental outcomes among children after CPB surgery. ${ }^{23}$ Newburger and colleagues $^{23}$ reported that among 8-year old children who had undergone the arterial switch operation in infancy, both longer ICU LOS and longer hospital LOS were associated with lower full-scale intelligence quotient and verbal intelligence quotient even after adjustment for perioperative and sociodemographic variables. In our study we found that each additional 30 days spent in the hospital was associated with a mean decrease in PDI of 6.6 points. The clinical outcome score, although its percent of variance (ie, $R^{2}$ ) was somewhat smaller than LOS, corresponded to a more dramatic drop in mean PDI of 10.9 for scores $\geq 3$.

Clinical scoring systems have been previously evaluated in children undergoing $\mathrm{CPB}$ surgery. Zobel and colleagues ${ }^{24}$ applied the Acute Physiologic Score for Children, Pediatric Risk of Mortality, Therapeutic Intervention Scoring System, and Organ System Failure score. All 4 scores differed significantly between survivors and nonsurvivors. ${ }^{24}$ However, that study was done during an earlier era (ie, 1989 to 1992) and did not evaluate neurodevelopmental outcomes. More recent studies have shown that the Risk Adjustment for Congenital Heart Surgery category has been validated as a risk factor for mortality in pediatric cardiac surgery, ${ }^{3}$ as has the modified sequential organ failure assessment score. ${ }^{4}$ The Aristotle Basic Complexity score predicts infants aged $<6$ months having longer ICU LOS. ${ }^{25}$ However, we are not aware of scoring systems that predict neurodevelopmental outcomes in this population.

The ideal surrogate outcome is inexpensive, easy to diagnose or observe, can be measured independent of treatment assignment, and is free of measurement error. The clinical outcome score that we developed does not incorporate any laboratory investigations and is therefore not associated with any cost. The components of the score (eg, time to first extubation) apply to all infants undergoing CPB surgery. The score is easy to calculate, and can be done before a patient's discharge from intensive care. Posting the derivation of the score (Table 1) in clinical settings (ie, ICU) would allow clinicians to quickly and accurately calculate the score for a given patient.

Our study has several limitations. Patients were operated on at a single surgical center, and therefore the validity of our findings should be confirmed in a multicenter study. 
For example, timing of sternal closure varies widely among centers. The sample size is relatively small and the "other group" $(\mathrm{n}=21)$ represents a heterogeneous group of lesions and surgical interventions. The timing of postoperative lactates was not prespecified but rather was at the discretion of the responsible intensivist. It is possible that patients who were more hemodynamically stable may have had less frequent lactate monitoring and therefore may have taken relatively longer to achieve a lactate level $\leq 2.0 \mathrm{mmol} / \mathrm{L}$ compared with patients who were deemed more hemodynamically unstable. Nonetheless we demonstrated a strong correlation between clinical outcome score and time to normalization of lactate. The long-term predictive ability of a PDI measured at age, 18 to 24 months is unclear. This is an observational study and therefore we can only establish an association between clinical score and PDI, not a cause-and-effect relationship. The clinical outcome score may not distinguish moderate from severely impaired brain function. In the multiple regression model it was not possible to adjust for ICU LOS, total hospital LOS, or duration of preoperative ventilation, because these variables are highly correlated with the clinical outcome score. For the same reason we were not able to control for single ventricle status in our multiple regression models. However, a separate analysis for the biventricular group indicated similar conclusions in terms of associations between clinical outcome score and neurodevelopmental outcomes (data not shown), reflecting the value of the clinical outcome score within the biventricular group. A separate analysis for the single ventricle group was not possible due to the small sample size. The Bayley III score has recently replaced the BSID-II, but at the time of study initiation we had a small sample of subjects who had completed a Bayley III. The results of this study cannot be generalized to the Bayley III, and therefore a similar study of patients who have completed a Bayley III assessment will be required to further validate the predictive ability of the clinical outcome score. Although the score is associated with adverse PDI score and with important perioperative clinical events such as time to normalization of lactate, we have not demonstrated that interventions aimed at lowering the score in any class of patients are associated with improvement in outcomes. By excluding subjects who died before a BSID-II assessment, we may have introduced bias in the relationship between clinical outcome score and postoperative clinical events, but this does not affect the demonstrated relationship between clinical outcome score and PDI score.

\section{CONCLUSIONS}

The novel clinical outcome score we describe was associated with adverse PDI score at age, 18 to 24 months and correlated strongly with other markers of postoperative events, including time to normalization of serum lactate and ICU and hospital LOS. This score is easy to calculate, has no cost, and can be used to identify children who may benefit from early and comprehensive neurodevelopmental intervention. The score is cardiac ICU-specific, and may be valuable as an outcome variable in future studies aimed at evaluating novel therapies in this high risk population.

\section{References}

1. Khairy P, Ionescu-Ittu R, Mackie AS, Abrahamowicz M, Pilote L, Marelli AJ. Changing mortality in congenital heart disease. J Am Coll Cardiol. 2010;56: 1149-57.

2. Boneva RS, Botto LD, Moore CA, Yang Q, Correa A, Erickson JD. Mortality associated with congenital heart defects in the United States: trends and racial disparities, 1979-1997. Circulation. 2001;103:2376-81.

3. Kang N, Cole T, Tsang V, Elliott M, de Leval M. Risk stratification in paediatric open-heart surgery. Eur J Cardiothorac Surg. 2004;26:3-11.

4. Shime N, Kageyama K, Ashida H, Tanaka Y. Application of modified sequential organ failure assessment score in children after cardiac surgery. $J$ Cardiothorac Vasc Anesth. 2001;15:463-8.

5. Mackie AS, Booth KL, Newburger JW, Gauvreau K, Huang SA, Laussen PC, et al. A randomized, double-blind, placebo-controlled pilot trial of triiodothyronine in neonatal heart surgery. J Thorac Cardiovasc Surg. 2005; 130:810-6.

6. Robertson CMT, Joffe AR, Sauve RS, Rebeyka IM, Phillipos EZ, Dyck JD, et al. Outcomes from an interprovincial program of newborn open heart surgery. $\mathrm{JPe}$ diatr. 2004;144:86-92.

7. Robertson CMT, Sauve RS, Joffe AR, Alton GY, Boddemann DM, Blakley PM, et al. The registry and follow-up of Complex Pediatric Therapies Program of Western Canada: a mechanism for service, audit, and research after life-saving therapies for young children. 2011;2011:965740. Epub May 8, 2011.

8. Bayley N. Bayley scales of infant development. San Antonio, TX: Psychological Corp; 1993.

9. Acton BV, Biggs WSG, Creighton DE, Penner KA, Switzer HN, Thomas JH, et al. Overestimating neurodevelopment using the Bayley-III after early complex cardiac surgery. Pediatrics. 2011;128:e794-800.

10. Wernovsky G, Wypij D, Jonas RA, Mayer JE Jr, Hanley FL, Wickey PR, et al. Postoperative course and hemodynamic profile after the arterial switch operation in neonates and infants. A comparison of low-flow cardiopulmonary bypass and circulatory arrest. Circulation. 1995;92:2226-35.

11. Cheung PY, Chui N, Joffe AR, Rebeyka IM. Robertson CM for the Western Canadian Pediatric Therapies Project Follow-Up Group. Postoperative lactate concentrations predict the outcome of infants aged 6 weeks or less after intracardiac surgery: a cohort follow-up to 18 months. J Thorac Cardiovasc Surg. 2005; 130:837-43

12. Karl TR, Hall S, Ford G, Kelly EA, Brizard CP, Mee RB, et al. Arterial switch with full-flow cardiopulmonary bypass and limited circulatory arrest: neurodevelopmental outcome. J Thorac Cardiovasc Surg. 2004;127:213-22.

13. Bellinger DC, Wypij D, duPlessis AJ, Rappaport LA, Jonas RA, Wernovsky G et al. Neurodevelopmental status at eight years in children with dextrotransposition of the great arteries: the Boston Circulatory Arrest Trial. J Thorac Cardiovasc Surg. 2003;126:1385-96.

14. McGrath E, Wypij D, Rappaport LA, Newburger JW, Bellinger DC. Prediction of IQ and achievement at age 8 years from neurodevelopmental status at age 1 year in children with d-transposition of the great arteries. Pediatrics. 2004;114:e572-6.

15. Creighton DE, Robertson CM, Sauve RS, Modemann DM, Alton GY, NettelAguirre A, et al. Neurocognitive, functional, and health outcomes at 5 years of age for children after complex cardiac surgery at 6 weeks of age or younger. Pediatrics. 2007;120:e478-86.

16. Tabbutt S, Nord AS, Jarvik GP, Bernbaum J, Wernovsky G, Gerdes M, et al. Neurodevelopmental outcomes after staged palliation for hypoplastic left heart syndrome. Pediatrics. 2008;121:476-83.

17. Atallah J, Dinu IA, Joffe AR, Robertson CM, Sauve RS, Dyck JD, et al. Two-year survival and mental and psychomotor outcomes after the Norwood procedure: an analysis of the modified Blalock-Taussig shunt and right ventricle-to-pulmonary artery shunt surgical eras. Circulation. 2008;118:1410-8.

18. Shime N, Kageyama K, Ashida H, Ueda M, Kitamura Y, Tanaka Y. Perioperative assessment of blood lactate levels in pediatric heart surgery. Jpn J Anesthesiol. 2001;50:752-7.

19. Duke T, Butt W, South M, Karl TR. Early markers of major adverse events in children after cardiac operations. J Thorac Cardiovasc Surg. 1997;114:1042-52. 
20. Hatherill M, Sajjanhar T, Tibby SM, Champion MP, Anderson D, Marsh MJ, et al. Serum lactate as a predictor of mortality after paediatric cardiac surgery. Arch Dis Child. 1997;77:235-8.

21. Trittenwein G, Nardi A, Pansi H, Golej J, Burda G, Hermon M, et al. Early postoperative prediction of cerebral damage after pediatric cardiac surgery. Ann Thorac Surg. 2003;76:576-80.

22. Gaies MG, Gurney JG, Yen AH, Napoli ML, Gajaraski RJ, Ohye RG, et al. Vasoactive-inotropic score as a predictor of morbidity and mortality in infants after cardiopulmonary bypass. Pediatr Crit Care Med. 2010;11:234-8.
23. Newburger JW, Wypij D, Bellinger DC, du Pleissis AJ, Kuban KC Rappaport LA, et al. Length of stay after infant heart surgery is related to cognitive outcome at age 8 years. J Pediatr. 2003;143:67-73.

24. Zobel G, Rodl S, Rigler B, Metzler H, Dacar D, Grubbauer HM, et al. Prospective evaluation of clinical scoring systems in infants and children with cardiopulmonary insufficiency after cardiac surgery. J Cardiovasc Surg. 1993;34:333-7.

25. Gillespie M, Kuijpers M, Van Rossem M, Ravishankar C, Gaynor JW, Spray T, et al. Determinants of intensive care unit length of stay for infants undergoing cardiac surgery. Congenit Heart Dis. 2006;1:152-60. 


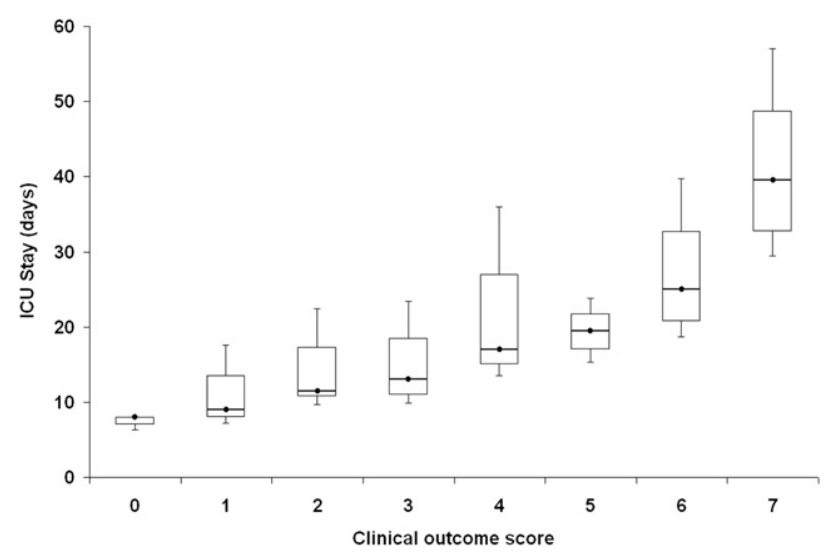

FIGURE E1. Relationship between postoperative intensive care unit $(I C U)$ length of stay and clinical outcome score $($ rho $=0.73 ; P<.0001)$.

TABLE E1. Psychomotor Developmental Index regression

\begin{tabular}{|c|c|c|c|c|}
\hline \multirow[b]{2}{*}{ Variable } & \multicolumn{2}{|c|}{ Univariate regression } & \multicolumn{2}{|c|}{ Multiple regression } \\
\hline & Effect $(95 \%$ CI $)$ & $P$ value & Effect size $(95 \%$ CI $)$ & $P$ value \\
\hline Antenatal diagnosis & $-4.10(-11.24,3.05)$ & .258 & & \\
\hline Sex & $2.04(-4.71,8.80)$ & .549 & & \\
\hline Birth weight (g) & $0.00(-0.00,0.01)$ & .149 & & \\
\hline Gestational age (wk) & $1.13(-1.31,3.58)$ & .360 & & \\
\hline Age at surgery (d) & $-0.39(-0.66,-0.11)$ & .006 & $-0.40(-0.68,-0.13)$ & .005 \\
\hline Duration of preoperative ventilation (d) & $-0.87(-1.34,-0.41)$ & .000 & & \\
\hline Year of surgery & $-1.26(-4.44,1.93)$ & .435 & & \\
\hline Duration of CPB (min) & $-0.05(-0.11,0.01)$ & .093 & & \\
\hline Duration of DHCA (min) & $-0.10(-0.37,0.17)$ & .449 & & \\
\hline Lowest flow $(\mathrm{mL} / \mathrm{kg} / \mathrm{min})(>10 \mathrm{~min})$ & $0.03(-0.06,0.11)$ & .557 & & \\
\hline Norwood/single ventricle & $-10.20(-17.17,-3.23)$ & .005 & & \\
\hline Total mother's education & $-0.01(-1.62,1.60)$ & .990 & & \\
\hline Clinical outcome score 1 vs 0 & $6.02(-3.12,15.16)$ & .194 & $-2.40(-13.78,8.98)$ & .676 \\
\hline Clinical outcome score 2 vs 0 & $6.45(-1.68,14.58)$ & .119 & $-3.54(-14.36,7.28)$ & .517 \\
\hline Clinical outcome score 3 vs 0 & $-9.69(-18.70,-0.68)$ & .035 & $-15.44(-26.79,-4.08)$ & .008 \\
\hline Clinical outcome score 4 vs 0 & $-3.51(-12.25,5.23)$ & .427 & $-10.98(-22.09,0.14)$ & .053 \\
\hline Clinical outcome score 5 vs 0 & $-0.06(-11.03,10.91)$ & .991 & $-10.78(-23.86,2.31)$ & .105 \\
\hline Clinical outcome score 6 or 7 vs 0 & $-11.35(-23.26,0.57)$ & .062 & $-16.08(-29.56,-2.61)$ & .020 \\
\hline
\end{tabular}

$C I$, Confidence interval; $C P B$, cardiopulmonary bypass; $D H C A$, deep hypothermic circulatory arrest. 
TABLE E2. Mental Developmental Index regression

\begin{tabular}{|c|c|c|c|c|}
\hline \multirow[b]{2}{*}{ Variable } & \multicolumn{2}{|c|}{ Univariate regression } & \multicolumn{2}{|c|}{ Multiple regression } \\
\hline & Effect $(95 \%$ CI) & $P$ value & Effect size $(95 \%$ CI $)$ & $P$ value \\
\hline Antenatal diagnosis & $0.60(-6.97,8.16)$ & .876 & & \\
\hline Sex & $3.94(-3.14,11.01)$ & .272 & & \\
\hline Birth weight (g) & $0.01(-0.00,0.01)$ & .058 & & \\
\hline Gestational age (wk) & $2.43(-0.10,4.97)$ & .060 & & \\
\hline Age at surgery (d) & $-0.46(-0.74,-0.17)$ & .002 & $-0.35(-0.64,-0.06)$ & .019 \\
\hline Duration of preoperative ventilation (d) & $-0.48(-0.99,0.03)$ & .067 & & \\
\hline Year of surgery & $2.47(-0.86,5.79)$ & .144 & & \\
\hline Duration of CPB (min) & $-0.03(-0.10,0.03)$ & .305 & & \\
\hline Duration of DHCA (min) & $-0.11(-0.41,0.19)$ & .453 & & \\
\hline Lowest flow (mL/kg/min) (>10 min) & $-0.04(-0.13,0.05)$ & .361 & & \\
\hline Norwood/single ventricle & $-1.70(-9.33,5.94)$ & .660 & & \\
\hline Total mother's education & $2.87(1.27,4.46)$ & .001 & $2.56(0.96,4.16)$ & .002 \\
\hline Clinical outcome score 1 vs 0 & $2.53(-7.15,12.22)$ & .605 & $-1.03(-12.76,10.69)$ & .861 \\
\hline Clinical outcome score 2 vs 0 & $4.36(-4.25,12.98)$ & .317 & $0.74(-10.43,11.91)$ & .896 \\
\hline Clinical outcome score 3 vs 0 & $-0.30(-9.99,9.40)$ & .952 & $-4.20(-15.94,7.53)$ & .479 \\
\hline Clinical outcome score 4 vs 0 & $-3.00(-12.20,6.20)$ & .520 & $-7.09(-18.56,4.38)$ & .223 \\
\hline Clinical outcome score 5 vs 0 & $5.88(-5.60,17.35)$ & .312 & $0.27(-13.23,13.78)$ & .968 \\
\hline Clinical outcome score 6 or 7 vs 0 & $-16.15(-28.48,-3.81)$ & .011 & $-13.25(-27.21,0.72)$ & .063 \\
\hline
\end{tabular}

$C I$, Confidence interval; $C P B$, cardiopulmonary bypass; $D H C A$, deep hypothermic circulatory arrest. 